Supporting Information for

\title{
An Electroosmotic Trap Against the Electrophoretic Force Near a Protein Nanopore Reveals Peptide Dynamics During Capture and Translocation
}

\author{
Alina Asandei ${ }^{1 \#}$, Irina Schiopu ${ }^{1 \#}$, Mauro Chinappi ${ }^{2 \#}$, Chang Ho Seo ${ }^{3}$, Yoonkyung Park ${ }^{4 *}$ and \\ Tudor Luchian ${ }^{5 *}$
}

${ }^{1}$ Department of Interdisciplinary Research, Alexandru I. Cuza University, Iasi, Romania

${ }^{2}$ Center for Life Nano Science@Sapienza, Istituto Italiano di Tecnologia, Roma, Viale Regina Elena 291, 00161, Italy

${ }^{3}$ Department of Bioinformatics, Kongju National University, Kongju, South Korea

${ }^{4}$ Department of Biomedical Science and Research Center for Proteineous Materials, Chosun University, Gwangju, South Korea

${ }^{5}$ Department of Physics, Alexandru I. Cuza University, Iasi, Romania

${ }^{\#}$ These authors contributed equally to this work

*Corresponding Authors: Yoonkyung Park (y_k_park@Chosun.ac.kr) and Tudor Luchian (luchian@uaic.ro) 

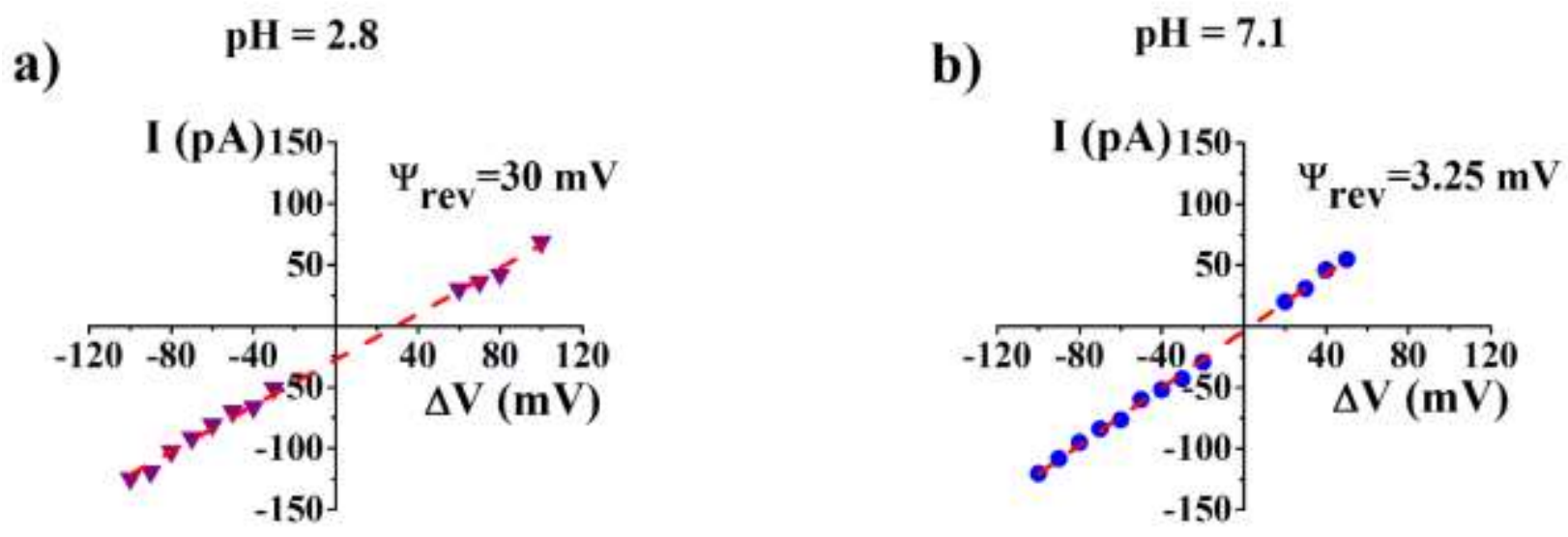

Fig. S1 Representative I- $\Delta \mathrm{V}$ diagrams illustrating the ionic current at various transmembrane potentials through a single $\alpha$-HL nanopore exposed to a salt gradient of $3 \mathrm{M} \mathrm{KCl}(\operatorname{trans}) / / 0.1 \mathrm{M}$ $\mathrm{KCl}$ (cis), used to calculate the reversal potential $\left(\Psi_{\text {rev }}\right)$ at $\mathrm{pH}=2.8$ (panel a) and $\mathrm{pH}=7.1$ (panel b)
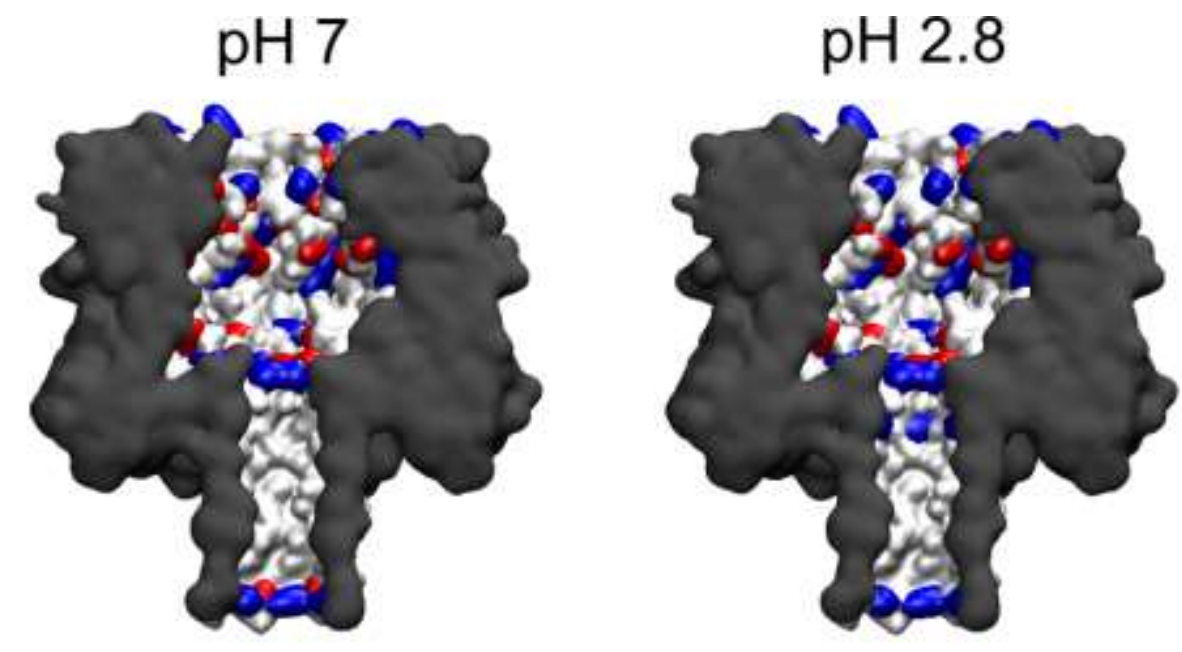

Fig. S2 Charged residues exposed toward the $\alpha$-HL's interior at $\mathrm{pH} 7$ and $\mathrm{pH} 2.8$. Red and blue correspond to negative and positive residues, respectively 


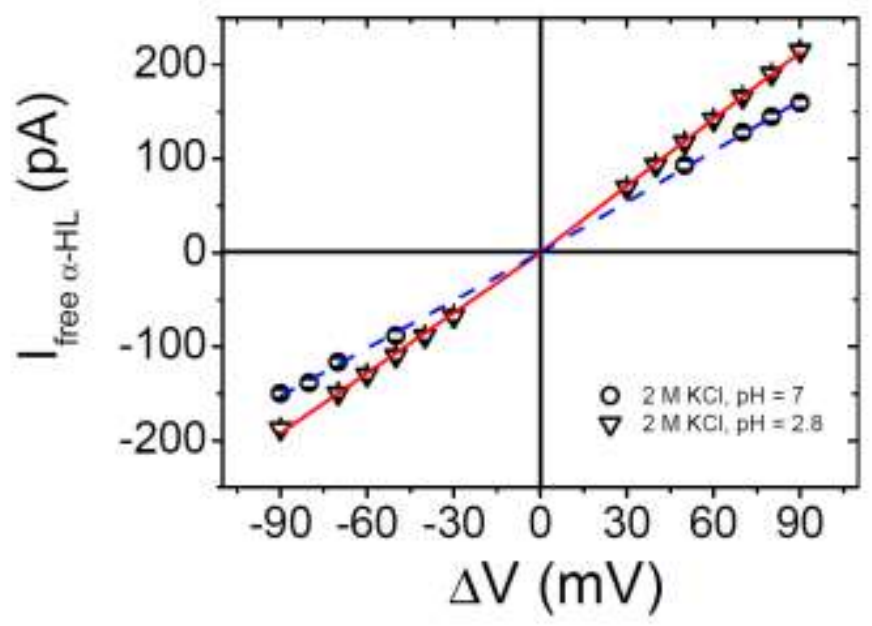

Fig. S3 Current-voltage (I- $\Delta \mathrm{V})$ diagrams of an open-pore $\alpha$-HL measured in neutral $(\mathrm{pH}=7)$ and acidic $(\mathrm{pH}=2.8)$, symmetrically added buffers $(2 \mathrm{M} \mathrm{KCl})$

a)

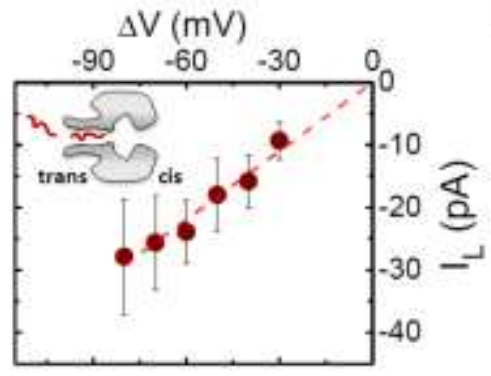

c)

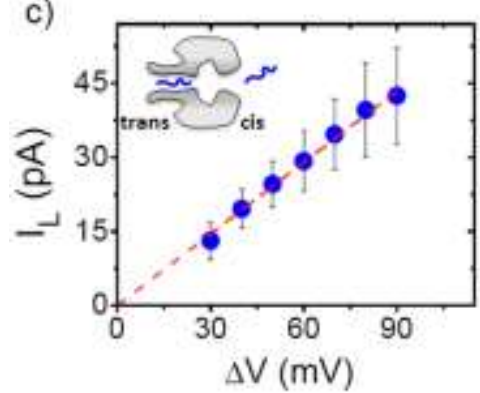

b)
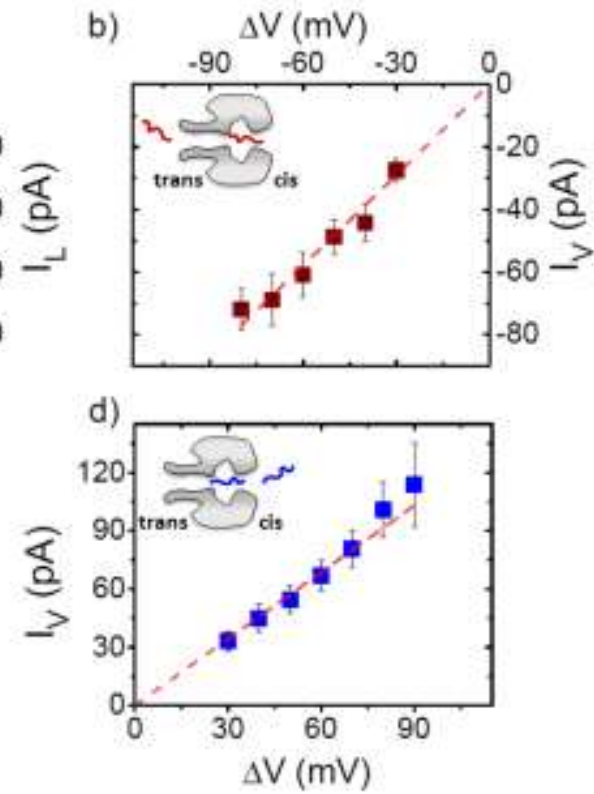

Fig. S4 Current-voltage (I- $\Delta \mathrm{V}$ ) diagrams of an $\alpha-\mathrm{HL}$ nanopore occupied by a single CAMA P6 peptide on the lumen $\left(\mathrm{I}_{\mathrm{L}}\right.$, panel a) or vestibule region $\left(\mathrm{I}_{\mathrm{V}}\right.$, panel $\left.\mathrm{b}\right)$, driven by the electroosmotic flow at negative $\Delta \mathrm{V}$ 's (peptide was added on the trans side). Panels $\mathrm{c}$ and d show similar (I- $\Delta \mathrm{V}$ ) diagrams for a $\alpha$-HL nanopore hosting a peptide on the lumen or vestibule region, respectively, 
driven into the pore from the addition side (cis side) under the influence of the electroosmotic flow.

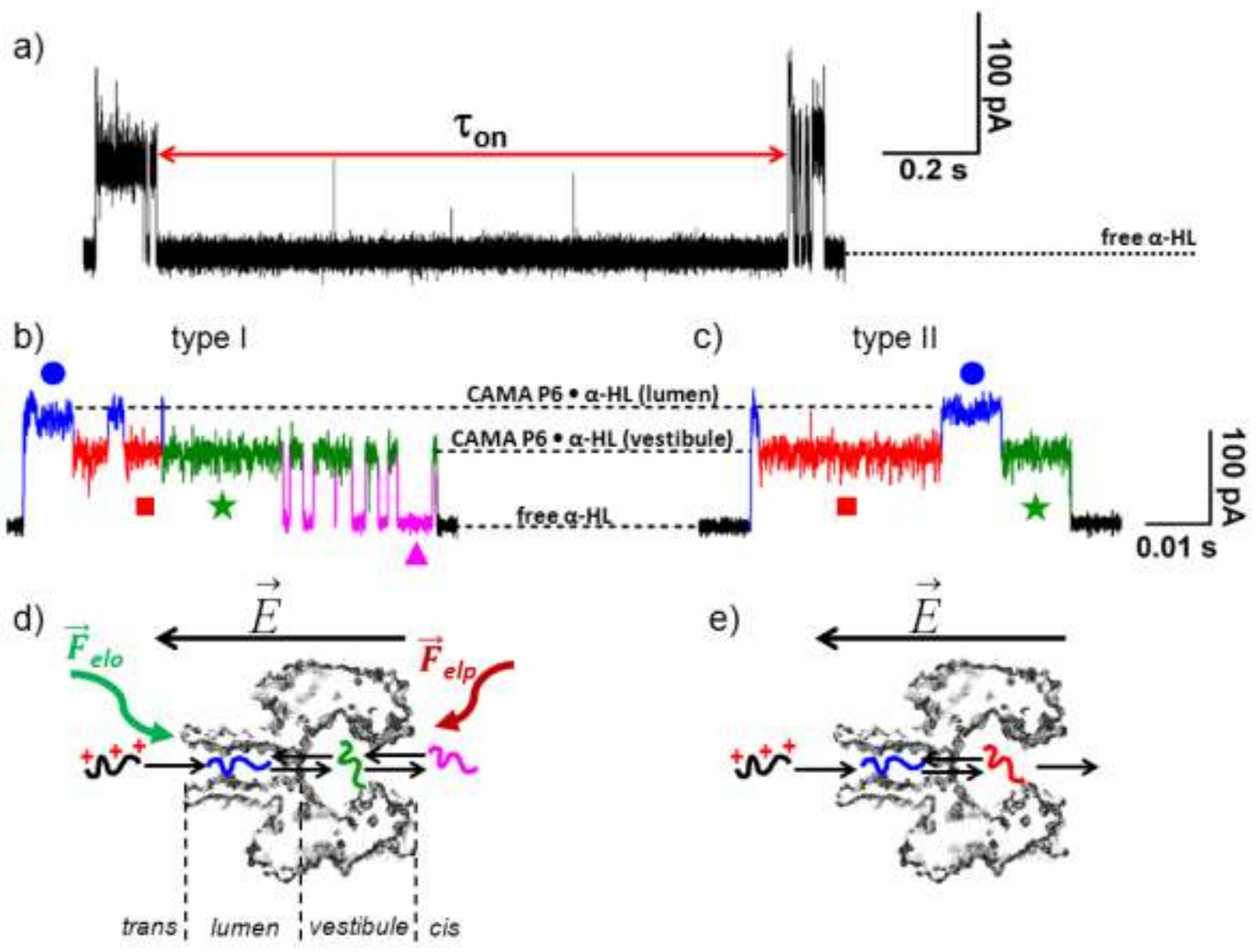

Fig. S5 Distinct microscopic sub-states characterizing the trans-added peptide dynamics inside the $\alpha$-HL nanopore, at low $\mathrm{pH}$. (a) Representative trace showing blockade events reflecting the cis-added, petide- $\alpha$-HL interactions at $\Delta \mathrm{V}=-80 \mathrm{mV}$, under the collective influence of electroosmotic $\left(\mathrm{F}_{\text {elo }}\right)$ and electrophoretic $\left(\mathrm{F}_{\text {elp }}\right)$ forces, at $\mathrm{pH}=2.8$. The open-pore current is denoted by 'free $\alpha-H L$ ', and the association time for peptide capture which separates consecutive blockade events is shown $\left(\tau_{\text {on }}\right)$. Peptide capture at the $\alpha$-HL's lumen and subsequent passage across the nanopore generate three distinct, brief current obstructions through the protein, upwardly oriented. In the first type of blockade events, a peptide captured at the $\alpha$-HL's lumen by the prevalent electroosmotic force, moves reversibly between the nanopore's lumen $(\bullet)$ and vestibule ( $\square$ ), as indicated by the corresponding current states in panel b. Eventually, the peptide moves from the lumen to the vestibule region ( $\star$ ), and exits the pore on the cis side $(\Delta)$, where it gets re-captured into the $\alpha$-HL's vestibule by the electrophoretic force. Another type of blockade events reflects the reversible movement of a peptide captured on the nanopore's lumen $(\bullet)$ to the $\alpha$-HL's vestibule ( $\square$ ), and then release from the vestibule $(\star)$ ) on the cis side, without re-capturing (panel c). The sketchy representation of molecular states and microscopic transitions associated to the type events described above, are shown in panels $\mathrm{d}$ and $\mathrm{e}$. 
a)

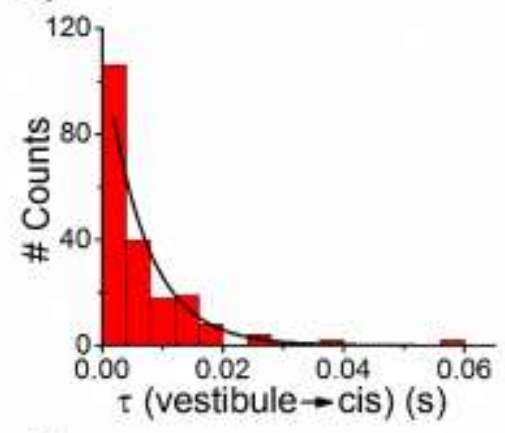

c)

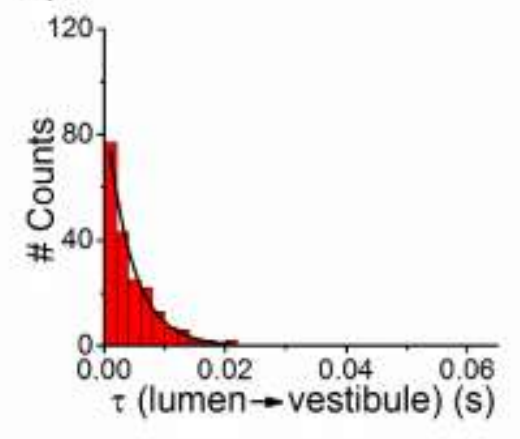

b)

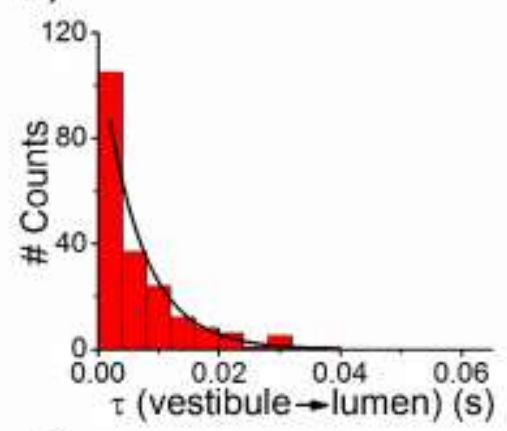

d)

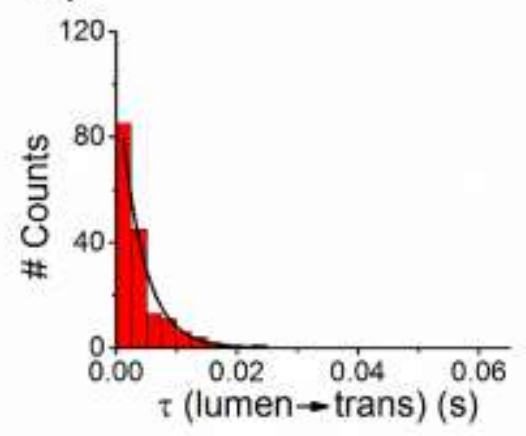

Fig. S6 Representative histograms of time intervals associated to the transitions made by a peptide inside the $\alpha$-HL nanopore, collected at $\mathrm{pH}=2.8$. The applied transmembrane potential was $\Delta \mathrm{V}=+80 \mathrm{mV}$. The dotted line represent the non-linear fit with a decaying monoexponential function $\left(\mathrm{y} \sim \exp \left(-\mathrm{t} / \tau_{\text {off }}\right)\right)$. 


\section{Determination of the protonated residues at $\mathrm{pH} 2.8$}

The $\alpha$-HL structure (PDB_ID:7AHL) ${ }^{1}$ was downloaded from OPM database ${ }^{2}$ and then loaded on the $\mathrm{H}++$ server $^{3}$ setting the salinity at $2 \mathrm{M}$. The server provided a $\mathrm{pK}_{\mathrm{a}}$ value for each single amino acid of each one of the seven chains. The $\mathrm{pK}_{\mathrm{a}}$ averaged over the seven chains are reported in the Table S1 for the titratable residues Aspartic acid (ASP), glutamic acid (GLU) and histidine (HIS). The residues that are protonated in the $\mathrm{pH} 2.8$ simulations are highlighted in gray.

Table S1 Average pKa for ASP, GLU and HIS residues of $\alpha-\mathrm{HL}$

\begin{tabular}{|l|r|r|}
\hline RES_TYPE & INDEX & pKa \\
\hline ASP & 2 & 0,386 \\
\hline ASP & 4 & 2 \\
\hline ASP & 13 & 3,21 \\
\hline ASP & 24 & 0 \\
\hline ASP & 29 & 1,257 \\
\hline ASP & 44 & 0,11 \\
\hline ASP & 45 & 0,82 \\
\hline ASP & 92 & 3,7 \\
\hline ASP & 100 & 0 \\
\hline ASP & 108 & 2,64 \\
\hline ASP & 127 & 2,86 \\
\hline ASP & 128 & 3,05 \\
\hline ASP & 152 & 0,038 \\
\hline ASP & 162 & 0,91 \\
\hline ASP & 183 & 0,9 \\
\hline ASP & 185 & 1,4 \\
\hline ASP & 208 & 3,04 \\
\hline ASP & 212 & 1,68 \\
\hline ASP & 227 & 1,67 \\
\hline ASP & 235 & 1,33 \\
\hline ASP & 246 & 2,44 \\
\hline ASP & 254 & 0 \\
\hline ASP & 255 & 2,6 \\
\hline ASP & 272 & 2,56 \\
\hline ASP & 276 & 0,08 \\
\hline ASP & 285 & 2,04 \\
\hline
\end{tabular}

\begin{tabular}{|l|r|r|}
\hline RES_TYPE & INDEX & pKa \\
\hline GLU & 31 & 4,25 \\
\hline GLU & 70 & 3,98 \\
\hline GLU & 71 & 3,82 \\
\hline GLU & 94 & 1,85 \\
\hline GLU & 111 & 2,52 \\
\hline GLU & 158 & 1,75 \\
\hline GLU & 250 & 1,4 \\
\hline GLU & 280 & 2,92 \\
\hline GLU & 287 & 4,1 \\
\hline GLU & 289 & 3,55 \\
\hline GLU & 290 & 3,8 \\
\hline & & \\
\hline HSE & 35 & 4,01 \\
\hline HSE & 48 & 3,56 \\
\hline HSE & 144 & 6 \\
\hline HSE & 259 & 5,5 \\
\hline
\end{tabular}




\section{Ions flows at $\mathbf{p H}=\mathbf{2 . 8}$ and $\mathrm{pH}=7$}

Molecular dynamics simulations allow to directly access to the contribution of $\mathrm{K}^{+}$and $\mathrm{Cl}^{-}$to the total current, dubbed $\mathrm{I}_{\mathrm{K}}$ and $\mathrm{I}_{\mathrm{Cl}}$ respectively. Table $\mathrm{S} 2$ and $\mathrm{S} 3$ reports the data for $\mathrm{pH}=2.8$ and $\mathrm{pH}=7$. It is apparent that $\mathrm{I}_{\mathrm{Cl}}$ is always larger than $\mathrm{I}_{\mathrm{K}}$.

Table S2 Ion currents for $\mathrm{pH}=2.8$ from MD simulations

\begin{tabular}{|c|c|c|c|}
\hline$\Delta \mathrm{V}$ & $\mathrm{I}_{\text {Tot }}$ & $\mathrm{I}_{\mathrm{K}}$ & $\mathrm{I}_{\mathrm{Cl}}$ \\
\hline-1.25 & $-2.89 \pm 0.06$ & $-0.76 \pm 0.09$ & $-2.13 \pm 0.09$ \\
\hline-0.625 & $-1.40 \pm 0.06$ & $-0.34 \pm 0.09$ & $-1.06 \pm 0.09$ \\
\hline-0.3125 & $-0.67 \pm 0.05$ & $-0.18 \pm 0.08$ & $-0.49 \pm 0.08$ \\
\hline 0.3125 & $0.81 \pm 0.05$ & $0.21 \pm 0.08$ & $0.60 \pm 0.08$ \\
\hline 0.625 & $1.60 \pm 0.06$ & $0.49 \pm 0.09$ & $1.11 \pm 0.09$ \\
\hline 1.25 & $2.24 \pm 0.09$ & $0.66 \pm 0.09$ & $1.58 \pm 0.09$ \\
\hline
\end{tabular}

Table S3 Ion currents for $\mathrm{pH}=7$ from MD simulations

\begin{tabular}{|c|c|c|c|}
\hline$\Delta \mathrm{V}$ & $\mathrm{I}_{\text {Tot }}$ & $\mathrm{I}_{\mathrm{K}}$ & $\mathrm{I}_{\mathrm{Cl}}$ \\
\hline-1.25 & $-1.63 \pm 0.09$ & $-0.53 \pm 0.12$ & $-1.10 \pm 0.13$ \\
\hline-0.625 & $-0.97 \pm 0.08$ & $-0.34 \pm 0.12$ & $-0.63 \pm 0.12$ \\
\hline-0.3125 & $-0.54 \pm 0.09$ & $-0.16 \pm 0.08$ & $-0.38 \pm 0.14$ \\
\hline 0.3125 & $0.84 \pm 0.14$ & $0.32 \pm 0.14$ & $0.52 \pm 0.14$ \\
\hline 0.625 & $1.81 \pm 0.09$ & $0.72 \pm 0.10$ & $1.09 \pm 0.12$ \\
\hline 1.25 & $3.80 \pm 0.09$ & $1.77 \pm 0.12$ & $2.03 \pm 0.13$ \\
\hline
\end{tabular}

\section{Theoretical framework for multistep translocations at positive $\Delta V$ 's}

We assume that the peptide can occupy only four distinct states, see Fig. S7:

- cis, the peptide is in the bulk at the cis side, close to the pore mouth. The current level is the open pore one since the peptide does not hinder the passage of the ions through $\alpha-\mathrm{HL}$ nanopore.

- vestibule, the peptide is in the vestibule of $\alpha$-HL nanopore (stuck at the pore entrance or in a bound state inside the vestibule). This state corresponds to a mild current reduction 
since the peptide only partially hinder the passage of ion and the pore constriction is not occupied by any residue.

- lumen, a portion of the peptide occupies the pore constriction (possibly more than one residues clog the constriction in a hairpin-like conformation as discussed in ${ }^{4-6}$ ). This state is associated to a strong current reduction.

- trans, the peptide is outside the nanopore on the trans side. The value of the current level is the one corresponding to the open pore and is the same as in cis state.

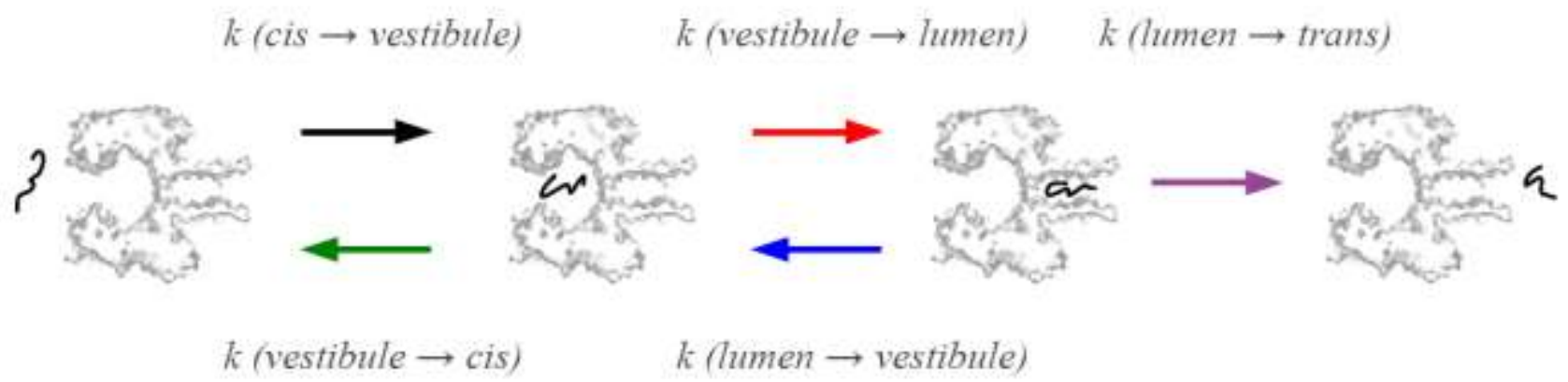

Fig. S7 Sketch of the four state model for peptide translocation.

We can model the peptide translocation as a pathway along these four states. The current traces collected during the experiments allowed the direct calculus of all the transition rates from one state to another. In particular, for two generic states A and B, indicated as:

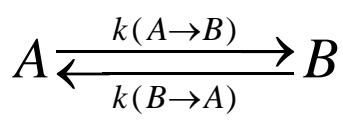

- $\quad M(A)$, the number of events where the system is in state A observed during the entire acquisition;

- $\quad M(B)$, the number of events where the system is in state $\mathrm{B}$ observed during the entire acquisition;

- $\tau_{A, i}$, the dwell time of the $\mathrm{i}$-th A events;

- $\tau_{B, i}$, the dwell time of the $\mathrm{i}$-th B events;

- $M(A \rightarrow B)$, the number of transition from the state $\mathrm{A}$ to the state $\mathrm{B}$ observed during the entire acquisition;

- $M(B \rightarrow A)$, the number of transition from the state $\mathrm{B}$ to the state $\mathrm{A}$ observed during the entire acquisition;

- $T_{A}=\sum_{i}^{M(A)} \tau_{A, i}$ the total time spent in the state A, regardless if the peptide goes toward cis or trans side;

- $T_{B}=\sum_{i}^{M(B)} \tau_{B, i}$ the total time spent in the state $\mathrm{B}$, regardless if the peptide goes toward cis or trans side.

Then we can get an estimation of the transition rate from a state $\mathrm{A}$ to a state $\mathrm{B}$ as: 


$$
k(A \rightarrow B)=\frac{M(A \rightarrow B)}{T_{A}} \quad \text { or } \quad k(B \rightarrow A)=\frac{M(B \rightarrow A)}{T_{B}}
$$

In principle, it is hence possible to estimate the rates corresponding to the transition between the four states (see Fig. S7). It is worth noting that, trans and cis states correspond to the same current levels so we cannot distinguish them and, consequently, we cannot calculate $T($ cis $)$ and $T$ (trans). However, the data indicates that for positive $\Delta \mathrm{V}$, the trans $\rightarrow$ lumen events are very rare, hence we can assume that the trans state is, in essence, an adsorbing state.

Thus, using eq. (2), we can estimate only the following four rates:

$$
\begin{aligned}
& k(\text { vestibule } \rightarrow \text { cis })=\frac{M(\text { vestibule } \rightarrow \text { cis })}{T_{V}} \\
& k(\text { vestibule } \rightarrow \text { lumen })=\frac{M(\text { vestibule } \rightarrow \text { lumen })}{T_{V}} \\
& k(\text { lumen } \rightarrow \text { vestibule })=\frac{M(\text { lumen } \rightarrow \text { vestibule })}{T_{L}} \\
& k(\text { lumen } \rightarrow \text { trans })=\frac{M(\text { lumen } \rightarrow \text { trans })}{T_{L}}
\end{aligned}
$$

\section{Rates and Free energies}

Given the stochastic nature of the translocation phenomena, it is often instrumental to represent the process in terms of free-energy profiles ${ }^{7}$. In an equilibrium process the free-energy profile $F$ as a function of a proper collective variable $\mathrm{z}$ is defined as the probability $p(z)$ to observe the corresponding state expressed in the natural logarithmic scale and in $k_{B} T_{m}$ units, i.e.,

$$
F(z)=-k_{B} T_{m} \ln p(z)
$$

where $\mathrm{k}_{\mathrm{B}}$ is the Boltzmann constant and $\mathrm{T}_{\mathrm{m}}$ is the absolute temperature.

In our case, we are not at equilibrium since an external forcing constantly acts on the system. However, our system is in a stationary state in a statistical sense, i.e. the dwell time probabilities distribution and the kinetic rates constant do not change in time. Hence we can employ eq. (7) to define a free-energy. The direct application of eq. (7) allows the estimation of the difference between the free-energy of the states vestibule and lumen as: 


$$
F(\text { lumen })-F(\text { vestibule })=-k_{B} T_{m} \ln \frac{T_{L}}{T_{V}}
$$

Free-energy barriers can be linked to the transition rates using phenomenological Arrhenius-like law such as:

$$
k(\text { lumen } \rightarrow \text { vestibule })=c \exp \left(-\frac{\Delta F(\text { lumen } \rightarrow \text { vestibule })}{k_{B} T_{m}}\right)
$$

The presence of the phenomenological constant $c$, does not allow us to estimate the free-energy barriers. The only quantities that can be deduced are the differences among the free-energy barriers that, assuming that the constant $c$ is identical for all the barriers, are:

$$
\begin{aligned}
& \Delta F(\text { vestibule } \rightarrow \text { lumen })-\Delta F(\text { vestibule } \rightarrow \text { cis })=-k_{B} T_{m} \ln \frac{k(\text { vestibule } \rightarrow \text { lumen })}{k(\text { vestibule } \rightarrow \text { cis })} \\
& \Delta F(\text { lumen } \rightarrow \text { vestibule })-\Delta F(\text { lumen } \rightarrow \text { trans })=-k_{B} T_{m} \ln \frac{k(\text { lumen } \rightarrow \text { vestibule })}{k(\text { lumen } \rightarrow \text { trans })}
\end{aligned}
$$

\section{References}

1. Song, L.; Hobaugh, M. R.; Shustak, C.; Cheley, S.; Bayley, H.; Gouaux, J. E. Structure of Staphylococcal $\alpha$-Hemolysin, a Heptameric Transmembrane Pore. Science 1996, 274, 1859-1865.

2. Lomize, M. A.; Lomize, A. L.; Pogozheva, I. D.; Mosberg, H. I. OPM: Orientations of Proteins in Membranes Database. Bioinformatics 2006, 22, 623-625.

3. Anandakrishnan, R.; Aguilar B.; Onufriev A. V. H++ 3.0: Automating pK Prediction and the Preparation of Biomolecular Structures for Atomistic Molecular Modeling and Simulations. Nucleic Acids Res. 2012, 40, 537-541.

4. Mereuta, L.; Roy, M.; Asandei, A.; Lee, J. K.; Park, Y.; Andricioaei, I.; Luchian, T. Slowing Down Single-Molecule Trafficking through a Protein Nanopore Reveals Intermediates for Peptide Translocation. Sci. Rep. 2014, 4, 3885.

5. Bonome, E. L.; Lepore, R.; Raimondo, D.; Cecconi, F.; Tramontano, A.; Chinappi, M. Multistep Current Signal in Protein Translocation through Graphene Nanopores. J. Phys. Chem. C 2015, 119, 5815-5823.

6. Di Marino, D.; Bonome, E. L.; Tramontano, A.; Chinappi, M. All-Atom Molecular Dynamics Simulation of Protein Translocation through an $\alpha$-Hemolysin Nanopore. $J$. Phys. Chem. Lett. 2015, 6, 2963-2968.

7. Rodriguez-Larrea, D.; Bayley, H. Protein Co-Translocational Unfolding Depends on the Direction of Pulling. Nat. Commun. 2014, 5, 4841. 